\title{
INTEGRAL REPRESENTATION WITHOUT ADDITIVITY
}

\author{
DAVID SCHMEIDLER ${ }^{1}$
}

\begin{abstract}
Let $I$ be a norm-continuous functional on the space $B$ of bounded $\Sigma$-measurable real valued functions on a set $S$, where $\Sigma$ is an algebra of subsets of $S$. Define a set function $v$ on $\Sigma$ by: $v(E)$ equals the value of $I$ at the indicator function of $E$. For each $a$ in $B$ let
\end{abstract}

$$
J(a)=\int_{-\infty}^{0}(v(a \geq \alpha)-v(S)) d \alpha+\int_{0}^{\infty} v(a \geq \alpha) d \alpha
$$

Then $I=J$ on $B$ if and only if $I(b+c)=I(b)+I(c)$ whenever $(b(s)-b(t))(c(s)-c(t)) \geq 0$ for all $s$ and $t$ in $S$.

1. Introduction. Let $\Sigma$ denote a nonempty algebra of subsets of a set $S$, let $B(S, \Sigma)$, or $B$ for short, denote the set of bounded, real valued, $\Sigma$-measurable functions on $S$, and let $v$ denote a monotonic real valued function on $\Sigma$ with $v(\phi)=$ 0 . Monotonicity means here that for any $E$ and $F$ in $\Sigma, E \subset F$ implies $v(E) \leq v(F)$. Since $S$ is in $\Sigma$, it is assumed without loss of generality that $v(S)=1$.

Choquet [1955] defined an integration operation with respect to the nonnecessarily additive set function $v$. Given a nonnegative valued function $a$ in $B$ let

$$
I(a)=\int_{S} a d v=\int_{0}^{\infty} v(\{s \in S \mid a(s) \geq \alpha\}) d \alpha
$$

where the integral on the right side is the extended Riemann integral. Choquet was interested in capacities and he imposed several additional restrictions on $\Sigma$ and on $v$. He dealt with a sigma algebra generated by the compact subsets of a metrisable compact topological space $S$, and with a strongly subadditive, and continuous at countable monotonic unions and intersections, set function $v$. These additional conditions are omitted here.

If every subset $E$ of $S$ is identified with its indicator function $E^{*}$, then the functional $I$ extends $v$ from $\Sigma$ to $B_{+}$the nonnegative functions in $B$. This extension is monotonic and positively homogeneous of degree one (i.e., $a \geq b$ on $S$ implies $I(a) \geq I(b)$ and $I(\lambda a)=\lambda I(a)$ for $\lambda>0)$. Two functions $a$ and $b$ in $B$ are said to be comonotonic if $(a(s)-a(t))(b(s)-b(t)) \geq 0$ for all $s$ and $t$ in $S$. Dellacherie $[\mathbf{1 9 7 0}]$ proved that $I(a+b)=I(a)+I(b)$ for comonotonic $a$ and $b$ in $B_{+}$. (There is a correctable mistake in his proof.)

In this paper we investigate the opposite direction, i.e., when a monotonic functional, say $I$, which is additive on comonotonic functions, can be represented

Received by the editors July 15, 1984 .

1980 Mathematics Subject Classification (1985 Revision). Primary 28C05; Secondary 47H07, $60 \mathrm{~A} 05,62 \mathrm{C} 10$.

${ }^{1}$ I am thankful to Jean-Francois Mertens for the reference to Choquet and to Dellacherie. Partial financial support from the Foerder Institute and NSF Grant No. SES-8026086 is gratefully acknowledged. Parts of this research were done at the Institute for Mathematics and its Applications at the University of Minnesota, and at the Department of Economics, University of Pennsylvania. 
through an integration operation as in (1) with respect to a monotonic set function $v$, defined by $v(E)=I\left(E^{*}\right)$ on $\Sigma$. Our motivation stems from the foundations of Bayesian decision theory and subjective probability. Within this framework an element $a$ in $B$ is interpreted as an uncertain payoff; $a(s)$ is the payoff or utility to the decision maker if the "state of nature" $s$ in $S$ occurs. The primitive concept is a binary relation over $B$, the decision maker's preferences between uncertain payoffs. Such a preference relation can be represented by a functional, say $I$, on $B$; i.e., $a$ stands in the binary relation to $b$ iff $I(a) \geq I(b)$. Formula (1) is interpreted as an expectation operation with respect to not necessarily additive probabilities.

The main result is stated and proved in the next section. $\S 3$ presents a variant of the main result useful for applications and a generalization to nonmonotonic set functions.

\section{The main result.}

THEOREM. Let $I: B \rightarrow R$ satisfying $I\left(S^{*}\right)=1$ be given. Suppose also that the functional I satisfies

(i) Comonotonic additivity. $a$ and $b$ comonotonic imply $I(a+b)=I(a)+I(b)$. (Hence $I(0)=0$.)

(ii) Monotonicity. $a \geq b$ on $S$ implies $I(a) \geq I(b)$. Then, defining $v(E)=I\left(E^{*}\right)$ on $\Sigma$ we have for all $a$ in $B$

$$
I(a)=\int_{0}^{\infty} v(a \geq \alpha) d \alpha+\int_{-\infty}^{0}(v(a \geq \alpha)-1) d \alpha .
$$

REMARK 1. A functional on $B$ satisfying comonotonic additivity and monotonicity also satisfies homogeneity (of degree 1). For a positive rational number $\alpha$, $I(\alpha a)=\alpha I(a)$ is implied by comonotonic additivity. The monotonicity assumption which is also a continuity assumption implies, in turn, the above equality for an arbitrary nonnegative number $\alpha$.

REMARK 2. The number 1 appearing in formula (2) stands for $v(s)=I\left(S^{*}\right)$. It is 1 only because of our normalization, which can be done w.l.o.g. because of homogeneity of $I$.

REMARK 3. The integrand in (2) can be referred to as the distribution of $a$ w.r.t. $v$. Formally we define for $a$ in $B$ its distribution $a^{*}: R \rightarrow R$ by

$$
a^{*}(\alpha)= \begin{cases}v(a \geq \alpha), & \alpha \geq 0 \\ v(a \geq \alpha)-v(S), & \alpha<0\end{cases}
$$

If $a$ is nonnegative then $a^{*}(\alpha)=0$ for all negative $\alpha$, and formulas (1) and (2) coincide. If $\theta$ is a negative lower bound of $a$ then $a^{*}(\alpha)=0$ for $\alpha \leq \theta$. If $\mu$ is an upper bound of $a$ then formula (2) is equivalent to $I(a)=\int_{\theta}^{\mu} a^{*}(\alpha) d \alpha$. We show now that (2) is implied by (1).

Since $a$ and any constant function are comonotonic we have

$$
I\left(a-\theta S^{*}\right)=I(a)+I\left(-\theta S^{*}\right) .
$$

On the other hand we claim that

$$
\int_{0}^{\mu-\theta}\left(a-\theta S^{*}\right)^{*}(\alpha) d \alpha=\int_{\theta}^{\mu} a^{*}(\beta) d \beta-\theta
$$


because $\left(a-\theta S^{*}\right)^{*}(\alpha)=v(a-\theta \geq \alpha)=v(a \geq \alpha+\theta)$, and denoting $\alpha+\theta=\beta$, $v(\alpha \geq \beta)=a^{*}(\beta)$ for $\beta \geq 0$ and $v(a \geq \beta)-1=a^{*}(\beta)$ for $\beta<0$. So, formula (2) is implied by formula (1).

PROOF OF THE THEOREM. As a conclusion of the remarks above it suffices to prove formula (1) for nonnegative functions. Suppose that (1) holds for all finite step functions and we prove it for an arbitrary nonnegative $\Sigma$-measurable function $a$ bounded by, say $\lambda$. For $n=1,2, \ldots$ and $1 \leq k \leq 2^{n}$ define $E_{n}^{k}=\{s \in$ $\left.S \mid \lambda(k-1) / 2^{n}<a(s) \leq \lambda k / 2^{n}\right\}$, and define $a_{n}(s)=\lambda(k-1) / 2^{n}$ and $b_{n}(s)=\lambda k / 2^{n}$ for $s$ in $E_{n}^{k}$. So

$$
a_{n}(s) \leq a_{n+1}(s) \leq a(s) \leq b_{n+1}(s) \leq b_{n}(s)
$$

for all $s$ and $n$. By monotonicity assumption $I\left(a_{n}\right) \leq I(a) \leq I\left(b_{n}\right)$ and by comonotonic additivity, $0 \leq I\left(b_{n}\right)-I\left(a_{n}\right)=\lambda / 2^{n} \rightarrow 0$ with $n \rightarrow \infty$. By our assumption about step functions $I\left(a_{n}\right)=\int_{0}^{\lambda} v\left(a_{n} \geq \alpha\right) d \alpha$ and $I\left(b_{n}\right)=\int_{0}^{\lambda} v\left(b_{n} \geq \alpha\right) d \alpha$ for all $n$. Hence in order to prove that $I(a)=\int_{0}^{\lambda} v(a \geq \alpha) d \alpha$ it is enough to show the inequality below for all $n$ :

$$
\int_{0}^{\lambda} v\left(a_{n} \geq \alpha\right) d \alpha \leq \int_{0}^{\lambda} v(a \geq \alpha) d \alpha \leq \int_{0}^{\lambda} v\left(b_{n} \geq \alpha\right) d \alpha .
$$

However this inequality follows from

$$
v\left(a_{n} \geq \alpha\right) \leq v(a \geq \alpha) \leq v\left(b_{n} \geq \alpha\right)
$$

which in turn follows from the monotonicity of $v$ and the definitions of $a_{n}$ and $b_{n}$ for $n=1,2, \ldots$.

PROOF FOR STEP FUNCTIONS. The proof is carried out by induction on the number of steps different from zero. Every nonnegative step function $a$ in $B$, which is not identically zero, has a unique representation $a=\sum_{i=1}^{k} \alpha_{i} E_{i}^{*}$ for some $k$ where $\alpha_{1}>\alpha_{2}>\cdots>\alpha_{k}>0$ and the sets $E_{i}, i=1, \ldots, k$, are pairwise disjoint and nonempty. For such a step function $a$ we have, defining $\alpha_{k+1}=0$,

$$
\int_{0}^{\alpha_{1}} v(a \geq \alpha) d \alpha=\sum_{i=1}^{k}\left(\alpha_{i}-\alpha_{i+1}\right) v\left(\sum_{j=1}^{i} E_{j}\right)
$$

The induction hypothesis is that for $k<n$

$$
I(a)=\sum_{i=1}^{k}\left(\alpha_{i}-\alpha_{i+1}\right) v\left(\sum_{j=1}^{i} E_{j}\right)
$$

and we prove it for $k=n$. Note that for $k=1$ it says $I\left(\alpha E^{*}\right)=\alpha v(E)$ which holds because of the homogeneity of $I$ and the definition of the set function $v$.

Given $a=\sum_{i=1}^{k} \alpha_{i} E_{i}^{*}$ as above, $a=b+c$ where $b=\sum_{i=1}^{k-1}\left(\alpha_{i}-\alpha_{k}\right) E_{i}^{*}$ and $c=\alpha_{k}\left(\sum_{i=1}^{k} E_{i}\right)^{*}$. By the induction hypothesis $(k-1<n)$,

$$
I(b)=\sum_{i=1}^{k-1}\left(\left(\alpha_{i}-\alpha_{k}\right)-\left(\alpha_{i+1}-\alpha_{k}\right)\right) v\left(\sum_{j=1}^{i} E_{j}\right)=\sum_{i=1}^{k-1}\left(\alpha_{i}-\alpha_{i+1}\right) v\left(\sum_{j=1}^{i} E_{j}\right)
$$

and $I(c)=\alpha_{k} v\left(\sum_{j=1}^{k} E_{j}\right)$. So, $I(b)+I(c)=\sum_{i=1}^{k}\left(\alpha_{i}-\alpha_{i+1}\right) v\left(\sum_{j=1}^{i} E_{j}\right)$. On the other hand, since $b$ and $c$ are comonotonic, $I(a)=I(b)+I(c)$ and formula (4) for $k=n$ has been proved. Q.E.D. 
REMARK 4. The opposite direction to the Theorem asserts that if a functional $I$ is defined by formula (2) with respect to some monotonic set function, then it satisfies conditions (i) and (ii) of the Theorem. The proof of this direction is quite easily obtained by reversing the proof of the Theorem.

More specifically: If a functional $I$ on $B(S, \Sigma)$ is defined by (2) for some monotonic $v$ one has to prove that it is comonotonically additive and monotonic. Monotonicity of $I$ is obvious since, $a \geq b$ on $S$ implies $a^{*} \geq b^{*}$ on $R$.

Comonotonic additivity is first shown for finite step functions in $B$. To see this and for future reference two immediate claims are stated without proofs.

Claim 1. Two finite step functions $b$ and $c$ in $B(S, \Sigma)$ are comonotonic iff there are an integer $k$, a partition of $S$ into $k$ pairwise disjoint elements of $\Sigma,\left(E_{i}\right)_{i=1}^{k}$ and two $k$-lists of numbers $\beta_{1} \geq \beta_{2} \geq \cdots \geq \beta_{k}$ and $\gamma_{1} \geq \gamma_{2} \geq \cdots \geq \gamma_{k}$ such that $b=\sum_{i=1}^{k} \beta_{i} E_{i}^{*}$ and $c=\sum_{i=1}^{k} \gamma_{i} E_{i}^{*}$.

Claim 2. Let $\left(E_{i}\right)_{i=1}^{k}$ be a $\Sigma$-measurable finite partition of $S\left(i \neq j\right.$ then $E_{i} E_{j}=$ $\phi)$, and let $a=\sum_{i=1}^{k} \alpha_{i} E_{i}^{*}$ with $\alpha_{1} \geq \alpha_{2} \geq \cdots \geq \alpha_{k}$. Then for any set function $v: \Sigma \rightarrow R$ with $v(\phi)=0$

$$
\int_{-\infty}^{\infty} a_{v}^{*}(\alpha) d \alpha=\sum_{i=1}^{k}\left(\alpha_{i}-\alpha_{i+1}\right) v\left(\sum_{j=1}^{i} E_{j}\right) .
$$

(The distribution of $a$ w.r.t. $v, a^{*}$, is defined in Remark 3 above and $\alpha_{k=1}=0$.)

Clearly if $I(a)$ is defined by the left side of (5) for finite step functions, then (5) and Claim 1 imply additivity for comonotonic finite step functions. The extension to any comonotonic functions in $B$ is done as usual by computing appropriate limits.

REMARK 5 . It is obvious that the Theorem and its converse hold if $B$ is substituted with $B_{0}$, the set of all finite step functions in $B$.

REMARK 6. If $I: B_{0} \rightarrow R$ satisfies comonotonic additivity and monotonicity it has a unique extension to all of $B$ which satisfies comonotonic additivity and monotonicity on $B$.

The proof is immediate since $B$ is the (sup) norm closure of $B_{0}$ in $R^{S}$ (see Dunford and Schwartz [1957, p. 240]), and monotonicity implies norm continuity.

3. Extensions. Let $B(S, \Sigma, K), B(K)$ for short, denote the set of functions in $B$ with values in $K \subset R$, and suppose that $[-1,1] \subset K$ and $K$ convex. A corollary to the Theorem is now stated. It is applicable to the neo-Bayesian model (Schmeidler [1984]).

COROLLARY. Let $I: B(K) \rightarrow R$ be given such that

(i) for all $\lambda$ in $K: I\left(\lambda S^{*}\right)=\lambda$,

(ii) if $a, b$ and $c$ are pairwise comonotonic, $0<\alpha<1$ and $I(a)>I(b)$, then $I(\alpha a+(1-\alpha) c)>I(\alpha b+(1-\alpha) c)$,

(iii) if $a \geq b$ on $S$ then $I(a) \geq I(b)$.

Then, defining $v(E)=I\left(E^{*}\right)$ on $\Sigma$, we have for all $a$ in $B(K)$, as in (2),

$$
I(a)=\int_{-\infty}^{0}(v(a>\alpha)-1) d \alpha+\int_{0}^{\infty} v(a>\alpha) d \alpha .
$$

Proof. The idea of the proof is to extend $I$ to all of $B$ and to show that the conditions of the Theorem are satisfied. 
Because of (i) $I$ is homogeneous on $B(K)$ and, by homogeneity, it can be uniquely extended to a homogeneous functional on $B$. The extension is also denoted by $I$. Once again by homogeneity (and by (iii)) the extended functional $I$ satisfies (iii), i.e. monotonicity on all of $B$. Comonotonic additivity of $I$ on $B$ is implied by the following Lemma and, it goes without saying, by homogeneity.

LEMMA. Given the conditions of the Corollary, let $a$ and $b$ in $B(K)$ be comonotonic with values in $[-1+\varepsilon, 1-\varepsilon]$ for some $\varepsilon>0$ and let $0<\lambda<1$. Then $I(\lambda a+(1-\lambda) b)=\lambda I(a)+(1-\lambda) I(b)$.

Proof. Denote $I(a)=\alpha$ and $I(b)=\beta$. By a condition of the Lemma, and by (i) and (iii) (of the Corollary), $\alpha S^{*}$ and $\beta S^{*}$ are in $B(k), I\left(\alpha S^{*}\right)=\alpha$ and $I\left(\beta S^{*}\right)=\beta$.

We have to prove that $I(\lambda a+(1-\lambda) b)=\lambda \alpha+(1-\lambda) \beta$. Suppose, per absurdum, that $I(\lambda a+(1-\lambda) b)>\lambda \alpha+(1-\lambda) \beta$. (The case of the other inequality is treated similarly.)

Let $0<\delta<\varepsilon$. Then by (i), $I(a)<I\left((\alpha+\delta) S^{*}\right)$ and $I(b)<I\left((\beta+\delta) S^{*}\right)$. Now,

$$
\begin{gathered}
\lambda \alpha+(1-\lambda) \beta+\delta=I\left(\lambda(\alpha+\delta) S^{*}+(1-\lambda)(\beta+\delta) S^{*}\right) \\
>I\left(\lambda a+(1-\lambda)(\beta+\delta) S^{*}\right)>I(\lambda a+(1-\lambda) b) .
\end{gathered}
$$

The equality holds by (i) and each of the two inequalities is implied by (ii). Since the inequality above holds for any $\delta(0<\delta<\varepsilon)$, we get the required contradiction. Q.E.D.

REMARK 1. The Corollary holds if $B(K)$ is replaced by $B_{0}(K)$, the set of bounded, $\Sigma$-measurable finite step functions on $S$ with values in $K$. The same is true for the Lemma.

Proposition 1. Suppose that $I: B_{0} \rightarrow R$ satisfies comonotonic additivity and positive homogeneity of degree 1. Then, defining $v(E)=I\left(E^{*}\right)$ on $\Sigma$, we have for all $a$ in $B_{0}$

$$
I(a)=\int_{-\infty}^{0}(v(a \geq \alpha)-v(S)) d \alpha+\int_{0}^{\infty} v(a \geq \alpha) d \alpha .
$$

PROPOSITION 2. Suppose that $I: B \rightarrow R$ satisfies comonotonic additivity and norm continuity. Then (6) above holds for all $a$ in $B$.

PROOFS. Remark 3 in $\S 2$ and the proof for (finite) step functions do not use monotonicity of $I$ or $v$, except to obtain homogeneity of $I$, which is assumed in Proposition 1 and implied by the continuity assumed in Proposition 2. Hence Proposition 1 holds and in order to prove Proposition 2 we have to extend it from finite step functions to bounded functions in $B$. We cannot use directly the proof of the Theorem because it heavily relies on montonicity. However, because of Remark 3 we can restrict the proof to nonnegative functions.

So, let $a$ in $B$ be given with values in the interval $[0, \lambda]$.

First it is proved that the Riemann integral $\int_{0}^{\lambda} a^{*}(\alpha) d \alpha$ exists. Otherwise there is an $\bar{\varepsilon}>0$ such that for every $n$ there is a partition of $[0, \lambda]$ to $k_{n}$ intervals $\lambda=\lambda_{0}>\lambda_{1}>\lambda_{2}>\cdots>\lambda_{k n}=0$ with $\lambda_{i-1}-\lambda_{i}<1 / 2^{n}, \bar{\lambda}_{i}$ and $\underline{\lambda}_{i}$ in $\left.] \lambda_{i-1}, \lambda_{i}\right]$ and $a^{*}\left(\bar{\lambda}_{i}\right) \geq a^{*}\left(\underline{\lambda}_{i}\right)$ for all $i$ such that

$$
\sum_{i} a^{*}\left(\bar{\lambda}_{i}\right)\left(\lambda_{i-1}-\lambda_{i}\right)-\sum_{i} a^{*}\left(\underline{\lambda}_{i}\right)\left(\lambda_{i-1}\right)\left(\lambda_{i-1}-\lambda_{i}\right)>\bar{\varepsilon} .
$$


For every $n$ two step functions are defined on $S: a_{n}(s)=\underline{\lambda}_{i}$ and $b_{n}(s)=\bar{\lambda}_{i}$ for $s$ s.t. $a(s)$ is in $\left.] \lambda_{i-1}, \lambda_{i}\right]$ for $i=1,2, \ldots, k_{n}$. Hence $a_{n}(s)-a(s) \leq 2 / 2^{n}$ and $b_{n}(s)-a(s)<2 / 2^{n}$ on $S$. Continuity of $I$ implies then that $I\left(a_{n}\right) \rightarrow I(a)$ and $I\left(b_{n}\right) \rightarrow I(a)$, that is $I\left(a_{n}\right)-I\left(b_{n}\right) \rightarrow 0$.

On the other hand by Proposition 1 and by definition of $a_{n}$

$$
I\left(a_{n}\right)=\int_{0}^{\lambda} v\left(a_{n} \geq \alpha\right) d \alpha=\sum_{i} a^{*}\left(\underline{\lambda}_{i}\right)\left(\lambda_{i-1}-\lambda_{i}\right)
$$

and similarly $I\left(b_{n}\right)=\int_{0}^{\lambda} v\left(b_{n} \geq \alpha\right) d \alpha=\sum_{i} a^{*}\left(\bar{\lambda}^{i}\right)\left(\lambda_{i-1}-\lambda_{i}\right)$. Thus $\mid I\left(a_{n}\right)-$ $I\left(b_{n}\right) \mid>\bar{\varepsilon}$ for all $n$, a contradiction.

As a conclusion we get that $\int_{0}^{\lambda} a^{*}(\alpha) d \alpha$ exists. Furthermore, for every $\varepsilon>0$ there is $n_{\varepsilon}$ such that for all $n>n_{\varepsilon}:\left|\sum_{i} a^{*}\left(\underline{\lambda}_{i}\right)\left(\lambda_{i-1}-\lambda_{i}\right)-\int_{0}^{\lambda} a^{*}(\alpha) d \alpha\right|<\varepsilon$, i.e. $\left|I\left(a_{n}\right)-\int_{0}^{\lambda} a^{*}(\alpha) d \alpha\right|<\varepsilon$. Since continuity of $I$ implies $I\left(a_{n}\right) \rightarrow I(a), I(a)=$ $\int_{0}^{\lambda} a^{*}(\alpha) d \alpha$. Q.E.D.

An additional result is needed for applications. It characterizes a subclass of functionals on $B$. A monotonic real valued function $v$ on $\Sigma$ with $v(\phi)=0$ is said to be convex (or strongly supperadditive) if for all $E$ and $F$ in $\Sigma: v(E)+v(F) \leq$ $v(E F)+v(E+F)$. The core of $v$ is, by definition, the set $\{p: \Sigma \rightarrow R \mid p(S)=v(S)$, $p$ additive and for all $E$ in $\Sigma, p(E) \geq v(E)\}$.

PROPOSITION 3. Suppose that $I: B \rightarrow R$ satisfies the conditions of the Theorem and $v$ on $\Sigma$ is defined as in the Theorem. Then the following three conditions are equivalent:

(i) $v$ is convex,

(ii) for all $a$ in $B: I(a)=\min \left\{\int a d p \mid p \in \operatorname{core}(v)\right\}$,

(iii) for all $b$ and $c$ in $B: I(b+c) \geq I(b)+I(c)$.

Proof. Suppose that $v$ is convex and let $a \in B_{0}$ be given. Then there exists a unique chain $\phi=E_{0} \subset E_{1} \subset E_{2} \subset \cdots \subset E_{k}=S$ with all the inclusions being strict and a unique list $\left(\alpha_{i}\right)_{i=1}^{k}$ with $\alpha_{i}>0$ for $i=1,2, \ldots, k-1$ s.t. $a=\sum_{i=1}^{k} \alpha_{i} E_{i}^{*}$. Clearly, by our Theorem, $I(a)=\sum_{i=1}^{k} \alpha_{i} v\left(E_{i}\right)$. Let $\Gamma$ be any finite subalgebra of $\Sigma$ with $E_{i}$ in $\Gamma$ for $i=1, \ldots, k$. Shapley $[\mathbf{1 9 6 5}]$ proved that there exists an additive real valued function $p$ on $\Gamma$ s.t. for all $E$ in $\Gamma, p(E) \geq v(E)$ and $p\left(E_{i}\right)=v\left(E_{i}\right)$ for $i=1,2, \ldots, k$. Hence, $\int a d p=I(a)$. By the Hahn-Banach theorem $p$ can be extended to all of $\Sigma$ s.t. $p \in \operatorname{core}(v)$. Since $\operatorname{core}(v)$ is compact in the weak star topology, (i) implies (ii) for all $a$ in $B$.

To prove that (ii) implies (iii) assume first that $b$ and $c$ are in $B_{0}$. Let $p$ in the core of $v$ be such that $I(a)=\int a d p$ where $a=b+c$. Using (7) again we get $I(b) \leq \int b d p$ and $I(c) \leq \int c d p$. But $\int a d p=\int b d p+\int c d p$ and the inequality in (iii) holds. Using, as previously, the compactness argument we obtain the implication for all $b$ and $c$ in $B$.

Finally (i) is a special case of (iii). Q.E.D.

Proposition 3 can also be proved by using Choquet's results for strongly subadditive $v$ (see Dellacherie $[\mathbf{1 9 7 0}]$ ). 


\section{REFERENCES}

G. Choquet (1955), Theory of capacities, Ann. Inst. Fourier (Grenoble) 5, 131-295.

C. Dellacherie (1970), Quelques commentaires sur les prolongements de capacités, Séminaire Probabilités V, Strasbourg, Lecture Notes in Math., vol. 191, Springer-Verlag, Berlin and New York.

N. Dunford and J. T. Schwartz (1957), Linear operators. Part I, Interscience, New York.

D. Schmeidler (1984), Subjective probability and expected utility without additivity (previous version (1982), Subjective probability without additivity), Foerder Inst. Econ. Res., TelAviv Univ.

L. S. Shapley (1965), Notes on n-person games. VII: Cores of convex games, Rand Corp. R.M. Also (1971), Internat. J. Game Theory 1, 12-26, as Cores of convex games.

School of Mathematical Sciences, Tel-Aviv University, Tel-Aviv, IsRael 